\title{
GI-MS46-P11 | Current Status of Sample Exchange Robots at the Photon Factory Macromolecular Crystallography Beamlines
}

Hiraki, Masahiko (Mechanical Engineering Center, Applied Research Laboratory, KEK, Tsukuba, JPN); Matsugaki, Naohiro (Structural Biology Research Center, Institute of Materials Structure Science, KEK, Tsukuba, JPN); Yamada, Yusuke (Structural Biology Research Center, Institute of Materials Structure Science, KEK, Tsukuba, JPN); Hikita, Masahide (Structural Biology Research Center, Institute of Materials Structure Science, KEK, Tsukuba, JPN); Senda, Toshiya (Structural Biology Research Center, Institute of Materials Structure Science, KEK, Tsukuba, JPN)

Structural Biology Research Center of Institute of Materials Structure Science operates five macromolecular crystallography beamlines at Photon Factory. We have developed sample exchange robots to achieve highthroughput, remote-controlled and/or fully-automated experiments. We developed and installed double tongs system that can grasp two cryo-pins simultaneously for rapid sample exchange on the robot PAM at beamlines BL5A, BL-17A, AR-NW12A and AR-NE3A. A beamline BL-1A was built for low energy SAD experiments and PAM was also installed. Because we covered a diffractometer with a helium chamber for effective low energy experiments, we developed another sample exchange robot PAM-HC. Only the Uni-Puck can be used for PAM-HC. We are developing a lid of PAM and PAM-HC. The lid consists of several doughnut shape plates and moves according to the movement of the robot arm. PAM can exchange cryo-pins for 10 seconds, but it takes 80 seconds for taking cryo-pin in and out a cassette under liquid nitrogen. This behavior is carried out in parallel with sample centering and diffraction experiment. Because fast detectors were installed in our all beamlines, a faster sample exchange robot is desired. Then with a cooperation of RIKEN and JASRI, we are developing a new sample exchange robot PAM3 having both features of double tongs of PAM and simple movement of PAM-HC. 